\title{
LISTA ACTUALIZADA Y ESTADO DE CONSERVACIÓN DE LOS MAMÍFEROS DEL PARQUE NACIONAL EL CHICO, HIDALGO, MÉXICO
}

\author{
S.D. HERnÁNDEZ-Flores \& A.E. RoJAS-MARTÍnez \\ Laboratorio de Ecología de Poblaciones, Centro de Investigaciones Biológicas, Universidad \\ Autónoma del Estado de Hidalgo. Carretera Pachuca-Tulancingo km 4.5 s/n, Colonia Carboneras, \\ Mineral de la Reforma, Hidalgo, MÉXICO. C.P. 42184. E-mail: danielhf83@hotmail.com y \\ aerojas@uaeh.edu.mx
}

Hernández-Flores, S.D. \& A.E. Rojas-Martínez. 2010. Lista actualizada y estado de conservación de los mamíferos del Parque Nacional El Chico, Hidalgo, México. Acta Zool. Mex. (n. s.), 26(3): 563-583.

RESUMEN. Se presenta la lista actualizada de los mamíferos del Parque Nacional El Chico con base en el trabajo de campo realizado entre los años 2004 y 2006. Se identificaron 30 especies, 13 de ellas se registran por primera vez en el parque: Glaucomys volans, Peromyscus melanotis, Reithrodontomys megalotis, $R$. sumichrasti, Spilogale gracilis, Cryptotis mexicana, Sorex saussurei, Dermanura azteca, Corynorhinus towsendii, Eptesicus fuscus, Lasiurus cinereus, Myotis californicus y M. velifer. El orden mejor representado, con 10 especies (33.33\%) fue Rodentia, seguido por Carnivora con ocho especies (26.67\%). El $16.67 \%$ de las especies están consideradas en estado de conservación delicado $(C$. mexicana, S. saussurei, Sciurus oculatus, G. volans y Corynorhinus mexicanus). Localmente las amenazas para la mastofauna son consecuencia de los asentamientos humanos y el turismo descontrolado. El Chico es importante para la conservación y manejo de los mamíferos de Hidalgo, porque en el habita el $23.8 \%$ de la mastofauna estatal, no obstante su reducida extensión territorial (2,739 ha).

Palabras clave: lista actualizada, mamíferos, estado de conservación, El Chico, Hidalgo.

Hernández-Flores, S.D. \& A.E. Rojas-Martínez. 2010. Check-list and conservation state of the mammals of National Park el Chico, Hidalgo, Mexico. Acta Zool. Mex. (n. s.), 26(3): 563-583.

ABSTRACT. We present a check-list of mammals of El Chico National Park based in field surveys realized between years 2004 to 2008 . The mammals identificated were 30 species and 11 are recorded for the first time in the park: Glaucomys volans, Peromyscus melanotis, Reithrodontomys megalotis, $R$. sumichrasti, Spilogale gracilis, Cryptotis mexicana, Sorex saussurei, Dermanura azteca, Corynorhinus towsendii, Eptesicus fuscus, Lasiurus cinereus, Myotis californicus y M. velifer. The most abundant species belonged to Rodentia and Carnivora. Threatened species account for $16.67 \%$ of the richness (C. mexicana, S. saussurei, Sciurus oculatus, G. volans y Corynorhinus mexicanus). Mammals in this park are threatened by human settlements and excessive tourism. El Chico National Park is important for conservation and management of mammals as $23.8 \%$ of the mammalian diversity of the State of Hidalgo.

Key words: check list, mammals, state of conservation, El Chico, Hidalgo.

Recibido: 02/12/2009; aceptado: 02/06/2010. 


\section{INTRODUCCIÓN}

Las Áreas Naturales Protegidas (ANP) tienen como objetivo principal asegurar la continuidad de los procesos ecológicos, mediante la conservación y aprovechamiento sostenible de la biodiversidad (Peña et al. 1998). A pesar de lo anterior la mayoría de las ANP de México carecen de información actualizada sobre los recursos biológicos que albergan, por tanto se desconocen las condiciones en las que se encuentran actualmente (Cervantes et al. 1995). Debido a la falta de información actualizada y confiable, los planes de manejo de las áreas protegidas podrían no garantizar la permanencia de las poblaciones de especies amenazadas, ni de la diversidad biológica en su conjunto (Álvarez del Toro 1985). Los mamíferos del Parque Nacional El Chico (PNEC) han sido poco estudiados, aun cuando este bosque ha sido considerado como área protegida por lo menos desde 1898 (INE-SEMARNAT 2000). Desde la década de los setentas existen trabajos que abordan el conocimiento de la mastofauna (Gallina et al. 1974; Córdova \& Rogel 1998), sin embargo incluyen muy poca información sobre los mamíferos voladores y para algunos animales no se confirmó su presencia en el parque. En la actualidad no existe un inventario actualizado y completo de los mamíferos que habitan el PNEC. Debido a lo anterior, el objetivo del presente trabajo fue elaborar una lista actualizada de los mamíferos que habitan en el PNEC, con el propósito de aportar información reciente, que sirva como base para proponer estrategias de manejo adecuadas para la conservación de estos animales.

\section{MATERIAL Y MÉTODOS}

El Parque Nacional El Chico se encuentra en la Sierra de Pachuca, ubicada en el Eje Neovolcánico Transversal y está relacionada con la Sierra Madre Oriental (Galindo et al. 1988; Zavala 1995). El PNEC se encuentra entre los $98^{\circ} 41^{\prime} 47^{\prime \prime}$ y $98^{\circ} 45^{\prime} 31^{\prime \prime}$ de longitud oeste y entre los $20^{\circ} 10^{\prime} 05^{\prime \prime}$ y $20^{\circ} 13$ ' $25^{\prime \prime}$ de latitud norte, $20 \mathrm{~km}$ al norte de la ciudad de Pachuca (Fig. 1) y tiene una extensión de 2,739 ha. Su territorio pertenece a los municipios de Mineral del Chico, Pachuca y Mineral del Monte (Villavicencio et al. 1992). El relieve del parque es complejo, contiene pendientes abruptas y escarpadas, franjas de escaso relieve y valles de considerable extensión, su altitud va desde los 2,350 hasta los 3,090 msnm. El clima es templado-húmedo con inviernos fríos, la temperatura media anual varía entre $10^{\circ}$ y $14^{\circ} \mathrm{C}$, con mínimas que llegan hasta $-9^{\circ} \mathrm{C}$ (Galindo et al. 1988). La precipitación media anual es de $1,567.9 \mathrm{~mm}$ (Gallina et al. 1974). La vegetación del parque está formada principalmente por bosque de oyamel (Abies religiosa) y bosque de oyamel-encino (Abies-Quercus), que cubren el 62.9 y el $10.55 \%$ de su superficie respectivamente, coexisten con otros tipos de vegetación de menor importancia como pastizales, bosque de encino, bosque pino-encino (Pinus-Quercus), bosque de táscate o tlaxcal (Juniperus) y bosque de cedro (Cupresus) (Soto et al. 2006). 


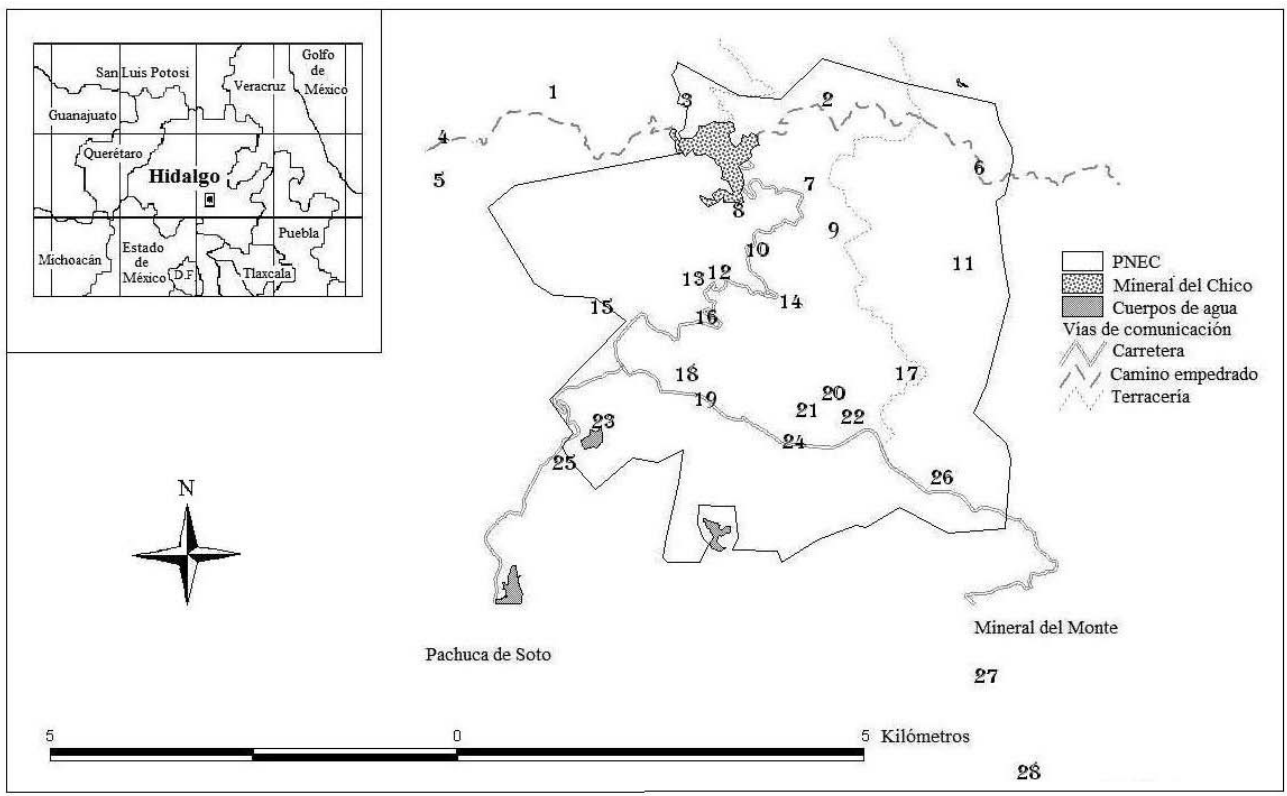

Figura 1. Ubicación del Parque Nacional El Chico. Los puntos numerados indican las localidades de colecta (los detalles de las localidades se muestra en el Cuadro 1)

Los animales fueron muestreados entre agosto del 2004 y agosto del 2006 (Cuadro 1, Fig. 1), en las cuatro estaciones del año y especialmente en los tipos de vegetación más importantes del parque. Para capturar roedores se utilizaron trampas tipo Sherman $(9 \times 8 \times 21 \mathrm{~cm})$, cebadas con una mezcla de hojuelas de avena, esencia de vainilla y crema de cacahuate, y se colocaron en forma de gradilla. Cada día fueron colocadas entre 45 y 50 trampas, separadas por una distancia de entre 5 y $10 \mathrm{~m}$. Las musarañas se colectaron mediante botes de plástico de un litro, enterrados completamente hasta que quedara la boca al nivel de la superficie del suelo y dispuestos en dos líneas de 10 botes cada una. Para la identificación de los ratones y las musarañas se consultó a Hall (1981) y Ceballos \& Galindo (1984). Los mamíferos medianos se muestrearon mediante rastros (heces y huellas), trampas tipo Tomahawk $(30 \times 30 \times 70 \mathrm{~cm})$, en total se colocaron 36 trampas durante todo el estudio (cebadas con sardina y manzana) y también se utilizaron trampas-cámara, marca Yashica T5D (análoga) con censor de movimiento utilizando el mismo cebo, se colocaron en cuatro sitios diferentes durante 10 días. Para completar el inventario se realizaron 15 entrevistas a personas de la zona, de entre 21 y 71 años de edad (apoyadas con ilustraciones) y se hicieron recorridos carreteros para recoger animales atropellados. La identificación de los rastros, así como de las observaciones y fotografías se hizo consultando guías de campo (Aranda 1981; 
Ceballos \& Galindo, 1984; Aranda 2000). Para capturar murciélagos se utilizaron redes de niebla de $6 \times 3 \mathrm{~m}$ y de $12 \times 3 \mathrm{~m}$, que se colocaron entre la vegetación, cerca de cuerpos de agua y en la entrada de minas abandonas. El muestreo concluyó con un total de 936 metros red, divididas en 14 noches $(66.8 \mathrm{~m} / \mathrm{red} /$ noche $)$ y en promedio las redes permanecieron abiertas 4.4 horas. La identificación se realizó consultando claves especializadas (Hall 1981; Álvarez et al. 1994; Medellín et al. 1997). Todos los ejemplares capturados fueron examinados in-situ y liberados posteriormente, únicamente fueron colectados un par de ejemplares para verificar su identificación. Los animales fueron depositados en la Colección de Mamíferos del Centro de Investigaciones Biológicas de la Universidad Autónoma del Estado de Hidalgo (UAEH-M). Finalmente, la identificación de los ejemplares fue corroborada mediante la consulta de la colección de mamíferos de la Universidad Autónoma Metropolitana, Unidad Iztapalapa (UAM-I).

El estado de conservación se presenta de acuerdo a la Norma Oficial Mexicana NOM-059-ECOL-2001 (SEMARNAT 2002), la lista roja de la IUCN (IUCN 2008) y se complementó con la información generada en el trabajo de campo acerca de su abundancia y la disponible en la literatura. Por último, con la finalidad de evaluar la calidad del inventario, se aplicaron estimadores de diversidad al esfuerzo invertido para capturar mamíferos pequeños no voladores y se utilizaron estimadores no paramétricos de riqueza de especies, basados en datos de presencia-ausencia: Ice, Jacknife 1 y Shao 2 (Moreno 2001). Para elaborar la lista taxonómica se aplicó la nomenclatura propuesta por Wilson \& Reeder (2005), con las excepciones señaladas por Ceballos et al. (2005). Las localidades mencionadas se muestran en el Cuadro 1 y la Figura 1. Se menciona el número total de ejemplares examinados por cada localidad, indicando si fueron liberados o depositados en la Colección Mastozoológica (UAEH-M), se indican las siglas de la colección, seguido por el número de catálogo. Las medidas somáticas se registran en el orden convencional (Hall 1981), en el caso de los murciélagos se adiciona la longitud del antebrazo (A). Cuando fueron examinados más de tres ejemplares para la misma especie se indica el promedio de sus medidas, así como la medida mínima y la máxima con sus valores entres paréntesis.

\section{RESULTADOS Y DISCUSIÓN}

Riqueza y Composición. Se registraron 30 especies de mamíferos silvestres, agrupadas en siete órdenes, 14 familias y 24 géneros (Anexo I, ver Lista Anotada más adelante). El orden con mayor número de especies fue Rodentia con 10 (33.33\% de las especies), seguido por los carnívoros con ocho especies que representan al $26.67 \%$ de los mamíferos del PNEC. Mephitis macroura y Spilogale gracilis se identificaron únicamente mediante entrevistas. La tuza (Thomomys umbrinus) no se capturó durante este trabajo, pero se consideró en el listado debido a que se revisó un 
Cuadro 1. Ubicación de las 28 localidades de colecta

1. Mina La Fortuna

2. La Compaña

3. Corredor de Truchas.

4. La Trucha Feliz

5. La Presa

6. Los Otates

7. Campamento Dos Aguas

8. Los Carpinteros

9. Peña El Cuervo

10. $600 \mathrm{~m}$ al Norte de Los Corrales

11. Campamento Conejos

12. Km 14 Carretera Mineral del Monte-

Mineral del Chico

13. Peña del Muerto

14. Los Corrales

15. Tlaxcalita

16. Km 13 Carretera Mineral del Monte-

Mineral del Chico

17. Los Magueycitos

18. Desarrollo Turístico La Gotera

19. Las Ventanas

20. La Chamusquina

21. $600 \mathrm{~m}$ al Norte del Campamento Los Cedros

22. Centro de Visitantes

23. Presa El Cedral

24. Campamento Los Cedros

25. La Estanzuela

26. Comisión Nacional Forestal

27. Km 2 Carretera Mineral del Monte-

Mineral del Chico

28. Km 1 Carretera Mineral del Monte-

Mineral del Chico
2,401

2,509

2,216

2,561

2,648

2,641

2,568

2,737

2,824

TC

2,700

BO,RN,TS,TT

2,920

$\mathrm{RC}$

2,805

2,758

TC

2,719

2,875

$\mathrm{RC}$

2,806

2,907

2,946

2,954

TC

2,997

2,995

2,980

RN,TS 2,785

3,013

RN,TC,TS,TT 2,762

RC 2,904

TS,TT

Las localidades están ordenadas de norte a sur, se especifica la metodología empleada y su altitud (BO: botes, CM: colecta manual, RC: recorrido carretero, RN: redes de niebla, TC: trampas cámara, TS: trampas Sherman y TT: trampas tomahawk). Cuando no se especifica la metodología empleada, la información se obtuvo por observaciones directas. 
ejemplar de 1992, depositado en la colección de mamíferos de la UAM-I. Las 30 especies registradas en el PNEC componen el $23.8 \%$ de la mastofauna del estado de Hidalgo (Ramírez-Pulido et al. 1986; Ramírez-Pulido \& Castro-Campillo 1990,1994; López-Wilchis \& López 1999; Ramírez-Pulido et al. 2000) y lo anterior resalta la importancia del parque en la conservación de los mamíferos, a pesar que su extensión representa menos del 0.14 \% del territorio estatal (Duran \& Larios 2001).

En este trabajo se adicionan 13 nuevas especies para el PNEC que pertenecen a cuatro órdenes diferentes: Rodentia (Glaucomys volans, Peromyscus melanotis, Reithrodontomys megalotis y R. sumichrasti), Carnivora (S. gracilis), Soricomorpha (Cryptotis mexicana y Sorex saussurei) y Chiroptera (Dermanura azteca, Corynorhinus towsendii, Eptesicus fuscus, Lasiurus cinereus, Myotis californicus y M. velifer; Anexo I).

Los mamíferos pequeños más abundantes fueron los ratones del género Peromyscus (ver Lista Anotada, $68.75 \%$ de los ejemplares examinados), siendo Peromyscus levipes el más abundante ( $78.79 \%$ de todos los Peromyscus colectados). La mayor abundancia de los ratones de este género se explica porque las trampas se colocaron mayormente al interior del bosque de oyamel, mismo que representa la comunidad vegetal más extensa en el parque, por lo que especies que están más asociadas a zonas abiertas con pastizales como Microtus mexicanus y $R$. megalotis se registraron con bajaa frecuencia (Ceballos \& Galindo 1984; González \& Cervantes 2005; Sánchez \& Oliva 2005).

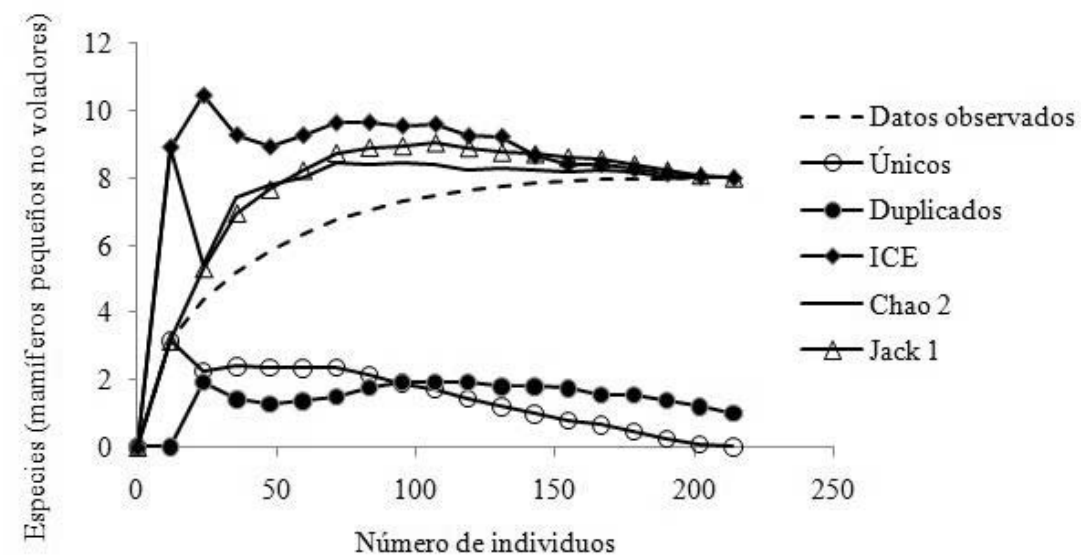

Figura 2. Especies de mamíferos pequeños no voladores esperadas y observadas, según los estimadores no paramétricos de la riqueza de especies y registros únicos y duplicados en el PNEC. 
Los mamíferos medianos más abundantes fueron Sciurus oculatus y Bassariscus astutus, hecho relevante en lo que respecta a $S$. oculatus, porque está incluida en la NOM-ECOL-059, es además endémica al Eje Neovolcánico y al parecer mantiene poblaciones estables en el parque, debido a que se puede observar en cualquier parte arbolada, coexiste con G. volans y Spermophilus variegatus, siendo la más común de las tres especies. El cacomixtle (B. astutus) fue el carnívoro más abundante de los mamíferos medianos que se encontraron muertos y también en las trampas cámara, donde constituyó el $74.5 \%$ de todos los registros fotográficos. La abundancia del cacomixtle en las trampas cámara probablemente se debe a que es una especie abundante y tolerante a las alteraciones humanas (S.D. Hernández-Flores obs. pers.)

El uso de las entrevistas permitió identificar especies medianas poco abundantes, que no se registraron por ningún otro método, este fue el caso de los zorrillos $M$. macroura y $S$. gracilis. Estas especies fueron consideradas presentes debido a que las personas entrevistadas las identificaron correctamente y además fueron registradas previamente por Morales-García (2007) en un bosque de pino-encino ubicado en el municipio aledaño de Huasca de Ocampo, que se encuentra a unos $20 \mathrm{~km}$ al sureste del parque, pero dentro de la misma cadena montañosa, denominada la Sierra de Pachuca.

Las especies de murciélagos colectadas fueron principalmente insectívoros (85.71\%), algo normal para un bosque templado a esta altitud (Cervantes et al. 1995) y sólo se identificaron tres especies mediante las redes de niebla (E. fuscus, M. californicus y $M$. velifer), donde $M$. velifer fue la especie más abundante. Otras especies como Corynorhinus mexicanus, $C$. towsendii y D. azteca únicamente se capturaron dentro de sus refugios.

Estimadores de riqueza de especies. La curva de acumulación de especies de los datos observados y los estimadores de riqueza de especies ICE, Jacknife 1 y Chao 2, calcularon en 8 especies la riqueza de mamíferos pequeños no voladores para el PNEC, lo que significa que se alcanzó un porcentaje de completitud del 100\%, con un esfuerzo de muestreo de 18 días y 852 trampas-noche (Fig. 2). Los resultados sugieren que el muestreo realizado para registrar a los mamíferos pequeños no voladores fue adecuado y que la probabilidad de encontrar especies nuevas mediante este método en el PNEC es baja.

Estado de Conservación. Es indudable que los mamíferos del PNEC son afectados por las actividades humanas y por lo menos tres especies (Puma concolor, Odocoileus virgnianus y Canis latrans) han sido extirpadas de la zona (Azcue et al. 1940; Gallina et al 1974; Córdova \& Rogel 1998; Galindo et al. 1988). Por medio de las entrevistadas realizadas en este trabajo se sabe que el venado cola blanca $(O$. virginianus) habitó en el parque hasta mediados del siglo pasado, incluso algunas de 
las personas encuestadas aún lo recuerdan en la zona, pero no se ha reportado en el parque por lo menos en los últimos 35 años (Cordova \& Rogel 1998; Galindo et al. 1988; Gallina et al. 1974: Villavcencio et al 1992), aún cuando la especie sobrevive de manera silvestre en otras regiones de la Sierra de Pachuca, en bosques de pino y de pino-encino en Huasca de Ocampo (Coronel-Arellano 2004, Morales-García 2007). El puma (P. concolor) también fue erradicado del parque, y existen registros históricos de la cacería de grandes gatos silvestres en los bosques de El Chico a mediados del siglo XVI (Azcue et al. 1940). No se sabe con precisión hace cuanto tiempo fue erradicado este felino de la zona, pero muy probablemente ocurrió varias décadas antes que el venado cola blanca, porque la gente sólo reconoce al gato montés o lince (Linx rufus) dentro de los bosques del PNEC.

Por último en esta lista encontramos al coyote (C. latrans), que anteriormente se ha reportado en El Chico (Gallina et al 1974, Villavicencio et al 1992), este carnívoro fue mencionado en las entrevistas, sin embargo no fue considerado en este trabajo debido a que no tuvimos ningún registro auditivo, ni de huellas o rastros de la especie durante el trabajo de campo. Por otra parte, las observaciones referidas por los pobladores fueron escasas y regularmente las ubicaron al menos 10 años antes de la realización de este trabajo.

El $16.67 \%$ de las especies del parque están catalogadas con un estado de conservación delicado (Anexo I), seis de ellas incluidas en la NOM-059-ECOL2001(C. mexicana, S. saussurei, S. oculatus y G. volans), mientras que C. mexicanus se encuentra en la lista roja de la IUCN. La IUCN considera que la tendencia poblacional para cinco especies que habitan el parque se encuentra en descenso, para 11 es considerada estable, en aumento para seis y para ocho especies se desconoce su tendencia poblacional (Anexo I).

Localmente existen diferentes amenazas para la mastofauna del PNEC, que directa o indirectamente son consecuencia de los asentamientos humanos y el turismo descontrolado. Para los mamíferos pequeños una amenaza constante son los incendios y la depredación por gatos y perros, en el muestreo con trampas-cámara más del $15 \%$ de las fotografías registradas fueron de estos animales, aun cuando se trabajó en zonas alejadas de los asentamientos humanos. Durante el trabajo de campo se observó que los perros hicieron presa de musarañas (C. mexicana) en más de una ocasión y fueron responsables de la muerte de un cacomixtle (B. astutus), hechos que probablemente reflejan el efecto de los carnívoros domésticos sobre los mamíferos silvestres. Los mamíferos medianos eventualmente son cazados por los habitantes de los poblados cercanos al parque, algunos por su piel como la zorra gris (Urocyon cinereoargenteus) y el lince (L. rufus) y otros por su carne como los armadillos, ardillas y zorrillos. En lo que se refiere a $L$. rufus, se sabe muy poco de esta especie en el parque, además de la fotografía tomada cerca de la localidad conocida como Los Corrales, sólo se conoce por los testimonios de la personas entrevistadas en el 
lugar. La escasez de este felino podría deberse a condiciones naturales, debido a que es una animal solitario y de hábitos nocturnos (Romero 2005), aunque es muy posible que las actividades humanas, como la cacería y la urbanización estén disminuyendo su número, tal como ha ocurrido en otras zonas del Eje Neovolcánico (Cervantes et al. 1995; Navarro-Frías et al. 2007). En lo que se refiere a los murciélagos, el principal problema se debe a la desconfianza y el temor que inspiran en la gente, son eliminados por los pobladores debido a la creencia errónea de que se alimentan de la sangre del ganado. Por otra parte los murciélagos que se refugian en minas son afectados por el vandalismo de los turistas en el parque. En todas las minas visitadas se encontraron evidencias de fogatas además de basura, por lo que es importante proteger estos refugios donde se resguardan por lo menos tres especies de murciélagos (C. mexicanus, C. towsendii y D. azteca). Si este problema no es controlado, los animales podrían abandonar los refugios temporal o permanentemente.

El PNEC es un área de gran importancia para la conservación de los mamíferos en el estado de Hidalgo, debido a que en este bosque protegido habita más de la mitad de los carnívoros reconocidos en el estado y cinco de sus especies se encuentran catalogadas con problemas de conservación. El estado de conservación de los mamíferos en el PNEC puede considerarse aceptable, sin embargo podría mejorar sustancialmente, reforzando la vigilancia e implementando programas de educación ambiental para los pobladores de la región y los turistas, enfocados a inculcar el respeto por los mamíferos de todas las especies.

\section{LISTA ANOTADA}

Orden Didelphimorphia

Familia Didelphidae

Didelphis virginiana (Kerr, 1792)

Ejemplares examinados. (3). Campamento Dos Aguas, 2,568 m (1), Comisión Nacional Forestal, 2,904 m, UAEH-M 60 (1), km 1 Carretera Mineral del MonteMineral del Chico, 2,801 m, UAEH-M 64 (1). Medidas somáticas. De un animal en taxidermia: LT:(670), C:(290), P:(40), O:(40). De dos machos: LT:700, C:290, P:61, O:43; peso 1,800 gr y LT:620, C:280, P:60, O:58; peso 870 gr. Observaciones. El animal examinado del Campamento Dos Aguas lo encontró muerto un guardaparque en noviembre del 2004 y se conserva en taxidermia en el Centro de Visitantes del PNEC, no fue posible determinar el sexo. Los dos machos fueron atropellados y colectados en los meses de octubre y noviembre del 2005 respectivamente. Los cráneos se encontraban muy fragmentados, aunque fue posible preparar la piel y limpiar el esqueleto, se encuentran depositados en la colección (UAEH-M). El tlacuache es un mamífero común en el PNEC, la mayoría de las personas entrevistadas lo reconocen dentro del parque y cerca de los poblados aledaños. 
Orden Xenarthra

Familia Dasypodidae

Dasypus novemcinctus (Linnaeus, 1758)

Ejemplares examinados. (1). Campamento Dos Aguas, 2,568 m. Medidas somáticas. De un macho: LT:715, C:313, P:87, O:38; peso 2,960 gr. Observaciones. Se examinó únicamente un ejemplar muerto, que recogieron los guardaparques cerca de la carretera, en agosto del 2006. El animal se conserva en taxidermia en el Centro de Visitantes del parque. Esta especie también se registró en una excreta de zorra gris, aunque el armadillo no se registró visualmente durante la realización del trabajo de campo, ni mediante las trampas cámara, la mayoría de las personas entrevistadas reconocen a este animal dentro del parque.

\section{Orden Insectivora}

Familia Soricidae

\section{Cryptotis mexicana (Coues, 1877)}

Ejemplares examinados. (5). Campamento Dos Aguas, 2,568 m (3), Campamento Conejos, 2,930 m. (1), $600 \mathrm{~m}$ al $\mathrm{N}$ del Campamento Los Cedros, 3,013 m (1). Medidas somáticas. De tres machos; LT:92, C:24, P:12, O:4; peso 5.5 gr, LT:93, C:25, P:12, O:3; peso 6 gr. y LT:91, C:22, P:11, O:5; peso 6 gr. Dos hembras; LT:100, C:27, P:12, O:4; peso 5.5 gr y LT:79, C:27, P:11, O:5; peso 5.5 gr. Observaciones. La especie es nuevo registro para el parque, está mencionada en el Plan de Manejo (Galindo et al. 1988), sin haber confirmado su presencia. Las dos musarañas del Campamento Dos Aguas se colectaron en enero del 2005, el macho fue donado por un guardaparque después de que un perro mató al animal. La hembra se capturó en una trampa de caída, mientras que el tercer ejemplar se recuperó del hocico de un perro en el campamento Dos Aguas. El ejemplar examinado del Campamento Conejos se colectó con una trampa Sherman, en abril del 2005 y el animal del Centro de Visitantes fue recolectado por el personal del parque.

Sorex saussurei (Merriam, 1892)

Ejemplares examinados. (1). Los Magueycitos, 2,907 m. Medidas somáticas. LT:100, C:34, P:11, O:9. Observaciones. Al igual que C. mexicana, esta musaraña es nuevo registro para el PNEC. De este único ejemplar examinado no fue posible determinar el sexo y tampoco se consideró el peso, porque se encontró muerto y en estado de descomposición. Esta musaraña también se registró en excrementos de zorra gris, cerca del campo Campamento Conejos a más de 2,900 m.

Orden Chiroptera

Familia Phyllostomidae

Dermanura azteca (Andersen, 1906)

Ejemplares examinados. (8). Mina La Fortuna, 2,401 m. Cuatro liberados y cuatro colectados (UAEH-M 54,55,56,57). Medidas somáticas. De ocho machos: LT:62.1 (54) (70), C:00 (00) (00), P:10.4 (8) (12), O:15.9 (14) (18), A:44.6 (44) (45); peso 21 
gr (17) (25). Observaciones. Es nuevo registro y es el único murciélago de hábitos alimentarios de tipo frugívoro del PNEC. Se ha reportado que esta especie forma colonias muy pequeñas y de los cuales se sabe muy poco (Ceballos \& Galindo, 1984). En la mina La Fortuna se observaron decenas de individuos en junio y septiembre del 2005 y en enero del 2006 se observaron murciélagos de esta especie en dos túneles que se encuentran sobre el río El Milagro en El Corredor de Truchas (Localidad 3), por lo que es posible sea una especie residente.

Familia Vespertilionidae

Corynorhinus mexicanus (Allen, 1916)

Ejemplares examinados. (1). Corredor de Truchas, 2,216 m (UAEH-M 9). Medidas somáticas. De una hembra: LT:92, C:42, P:09, O:30, A:40; peso 8 gr. Observaciones. Este murciélago se capturó en diciembre del 2004, el animal se encontraba perchado y muy aletargado a baja altura dentro del túnel de una mina y se colectó con facilidad de manera directa. La hembra estaba inactiva y presentaba grasa muscular y visceral. Aunque estos túneles fueron visitados en tres ocasiones más ya no se observaron murciélagos de esta especie. Es la única especie de murciélago que se había registrado con anterioridad en el parque (Córdova \& Rogel 1998).

Corynorhinus towsendii (Cooper, 1837)

Ejemplares examinados. (6). Corredor de Truchas, 2,216 m. Cinco liberados y uno sacrificado (UAEH-M 10). Medidas somáticas. De seis hembras: LT:102.3 (95) (107), C:45 (41) (48), P:9.8 (9) (11), O:34.5 (33) (36), A:43 (41) (45); peso 10.7 gr (9.5) (12). Observaciones. Esta especie es nuevo registro para el PNEC. Ninguno de estos animales se capturó en las redes, al igual que $C$. mexicanus, los ejemplares se colectaron en una mina cuando estaban perchados y muy aletargados en el interior del túnel. Corynorhinus towsendii a diferencia de C. mexicanus, se observó en la mina en los meses de diciembre del 2004, mayo del 2005 y enero del 2006. Todos los ejemplares examinados fueron hembras, pero ninguna se encontraba en estado reproductivo.

Eptesicus fuscus (Palisot de Beauvois, 1796)

Ejemplares examinados. (1). La Trucha Feliz, 2,557 m (UAEH-M 78). Medidas somáticas. De una hembra: LT:122, C:54, P:08, O:12, A:52; peso 22 gr. Observaciones. Este único ejemplar se capturó en septiembre del 2005, en la orilla de una presa que se encuentra en la Trucha Feliz y es el primer registro de la especie para el PNEC. La hembra se encontraba inactiva para la reproducción y presentaba abundante grasa muscular y visceral.

Laciurus cinereus (Palisot de Beauvois, 1796)

Ejemplares examinados. (1). El Cuervo, 2,557 m (UAEH-M 146). Medidas somáticas. De un macho LT:121, C:50, P:11, O:10, A:52; peso 19 gr. Observaciones. Este único ejemplar se colectó casualmente durante una visita al parque después de haber terminado el periodo de muestreo, el 5 de enero del 2008, a la orilla del camino 
hacia la peña del Cuervo, el animal se encontró muerto, aparentemente debido al frío, porque días previos nevó con intensidad como no ocurría desde hace varios años atrás.

Myotis californicus (Audubon \& Bachman, 1842)

Ejemplares examinados. (5). La Trucha Feliz, 2,884 m (2), Presa El Cedral, 2,785 m (3). Se sacrificaron todos los animales y están en proceso de catalogarse para la colección. Medidas somáticas. De cinco machos: LT:81.2 (77) (89), C:41.6 (36) (57), P:6.8 (6) (7), O:15 (11) (20), A:35.6 (33) (39); peso 4 gr (4) (4). Observaciones. La especie está citada en el Plan de Manejo (1988), suponiendo que estaba presente porque habita ambientes similares. Los animales aquí examinados son los primeros registros de $M$. californicus para el parque. Los cinco machos se capturaron cerca de cuerpos de agua. En estos animales se presentó el dicromatismo citado por Ceballos \& Galindo (1984). Dos de los ejemplares examinados presentaban la fase de coloración clara y el resto presentó la fase obscura. Esta especie se capturó justo después de oscurecer.

\section{Myotis velifer (Allen, 1890)}

Ejemplares examinados. (7). La Trucha Feliz 2,557 m (6), Presa El Cedral, 2,785 m (1). Tres liberados y cuatro sacrificados (UAEH-M 79,80,81,98). Medidas somáticas. De cuatro machos: LT:92.5 (89) (95), C:42.5 (38) (48), P:9.2 (8) (11), O:13.7 (13) (14), A:45 (44) (46); peso 9.6 gr (8) (12). Dos hembras: LT:98, C:40, P:11, O:14, A:43.5; peso 10 gr. y LT:93, C:44, P:9, O:10, A:45; peso 6 gr. Observaciones. La especie es nuevo registro para el PNEC. Los animales se colectaron en los meses de mayo y julio del 2005. En uno de los individuos no se observó con claridad la región de los omóplatos casi desprovista de pelo, que puede considerarse una característica diagnóstica para su identificación y citada por Medellín et al. (1997), sin embargo todas las demás características coinciden con las citadas por Hall (1981) para M. velifer.

\section{Orden Carnivora}

Familia Canidae

\section{Urocyon cinereoargenteus (Schreber, 1775)}

Ejemplares examinados. (1) Localidad desconocida. Medidas somáticas. De un animal en taxidermia LT (910), C (370), P (130), O (70). Observaciones. El único animal examinado se encuentra en el Centro de Visitantes. Se decomisó a la persona que lo cazó cuando intentó venderlo en Mineral del Chico, en noviembre del 2004, no fue posible sexarlo y se desconoce el lugar exacto dentro del parque en donde se colectó. La especie está bien distribuida en el parque, sus excrementos son comunes en los senderos así como en las zonas rocosas y elevadas. Algunos de estos lugares son: el Campamento Dos Aguas, Campamento Conejos, la zona incendiada en el km 13, Tlaxcalita, Las Ventanas, cerca de la presa El Cedral y La Chamusquina. También se tomaron fotografías de este animal mediante cámaras automáticas en dos 
localidades diferentes. En marzo del 2006 se tomaron cuatro fotografías en la localidad conocida como La Chamusquina, todas las fotografías se tomaron durante la noche, entre las 20:00 y 21:00 hrs. La segunda localidad en donde se registró la zorra gris mediante este método se ubica $600 \mathrm{~m}$ al Norte de Los Corrales, en este lugar únicamente se obtuvo un registro fotográfico a las 20:00 hrs el día 27 de abril del 2006. La zorra gris parece ser común y la mayoría de la gente que trabaja o vive en el parque y sus alrededores lo ha observado por lo menos en una ocasión en los últimos tres años.

\section{Familia Felidae}

Lynx rufus (Schreber, 1777)

Ejemplares examinados. Ninguno. Observaciones. Durante el estudio solamente se consiguió un registro fotográfico mediante una trampa-cámara, $600 \mathrm{~m}$ al $\mathrm{N}$ de los Corrales. Cerca del paraje conocido como Tlaxcalita, se colectó una excreta similar en tamaño y forma a las descritas para el gato montés (Aranda, 2000). El lince fue reconocido por 8 de las 15 personas entrevistadas y en todos los casos solamente lo han observado una vez en su vida. Algunas de estas observaciones son de 30 años atrás, aunque la mayoría son de los últimos 10 años y los testimonios más recientes son incluso del tiempo en que se realizó el presente trabajo. Este animal fue observado por la gente en regiones elevadas del parque, cercanas a la comunidad de Pueblo Nuevo, La Estanzuela, las Ventanas y en las Monjas, que se encuentran al norte del parque cerca de la comunidad de El Puente.

Familia Mustelidae

Mustela frenata (Lichtenstein, 1831)

Ejemplares examinados. Ninguno. Observaciones. Se registró un ejemplar muerto en abril del 2005, mediante fotografías proporcionadas por las autoridades del PNEC, cerca de Los Carpinteros. No fue posible recuperar al animal, lo que impidió sexarlo y determinar la causa por la que murió. La onza es común en el parque, la gente la reconoce dentro del bosque y más comúnmente en áreas abiertas cerca del Centro de Visitantes y en los poblados.

Familia Mephitidae

Conepatus leuconotus (Lichstenstein, 1832)

Ejemplares examinados. (1). La Compaña, 2,509 m. Observaciones. Esta especie se describió por primera vez en las cercanías de El Chico (Álvarez et al. 1997), sin embargo parece que en la actualidad el zorrillo de espalda blanca se ha vuelto raro en el área. Durante el desarrollo del presente estudio no fue posible capturar u observar ningún animal. El ejemplar examinado fue cazado en el año 2000 en La Compaña y únicamente se revisó la piel curtida, razón por la que no se consideraron las medidas. Esta especie no se registró mediante las entrevistas, aun cuando las personas encuestadas fueron elegidas por su relación con el parque y sus recursos. 
Mephitis macroura (Lichtenstein, 1832)

Ejemplares examinados. Ninguno. Observaciones. El zorrillo listado no fue observado ni capturado durante el trabajo de campo y solamente se registró mediante las entrevistas. Fue reconocido por ocho de las 15 personas encuestadas y la mayoría de los avistamientos ocurrieron en los últimos cinco años. Las localidades donde se le observó son cercanas a la comunidad de La Estanzuela, Pueblo Nuevo y los llanos aledaños al Centro de Visitantes.

\section{Spilogale gracilis (Linnaeus, 1758)}

Ejemplares examinados. Ninguno. Observaciones. Este pequeño zorrillo se registró solamente mediante entrevistas. Siete de las personas entrevistadas lo identificaron y cada una de ellas lo observó en una sola ocasión. En las entrevistas fue igualmente reconocido como M. macroura y se le observó cerca de las comunidades de La Estanzuela, Carboneras y en los alrededores de la Peña del Cuervo y Las Ventanas. Este zorrillo no se había registrado anteriormente en el parque y es considerado en este trabajo con base a la información de las entrevistas y porque al igual que M.macroura existen registros recientes de esta especie en bosques templados del municipio de Huasca de Ocampo, un lugar relativamente cercano a El Chico (Morales-García 2007).

\section{Familia Procyonidae}

Bassariscus astutus (Lichtenstein, 1830)

Ejemplares examinados. (3). La Peña del Muerto, $2,758 \mathrm{~m}$ (1), Centro de Visitantes, 2,980 m (1), Campamento Los Cedros, 2,995 m (1). Medidas somáticas. De un macho, LT:750, C:350, P:73, O:55; peso 1,670 gr. Una hembra, LT:758, C:334, P:70, O:49; peso 1,125 gr. De un animal en taxidermia, LT (760), C (350), P (60), O (40). Observaciones. El cacomixtle fue el carnívoro más abundante en el estudio. La hembra examinada fue colectada en marzo del 2005, cerca del Centro de Visitantes, presentaba heridas profundas en el abdomen y cerca de los hombros y por las dimensiones se concluyó que fue muerto por un perro. El macho se recogió a un lado de la carretera, cerca de la Peña del Muerto y presentaba un golpe cerca de la cabeza. El animal en taxidermia fue colectado y preparado por las autoridades del parque, pero se desconocen datos sobre el sexo y la condición reproductiva. El cacomixtle fue bien reconocido por las personas entrevistadas, en varios puntos dentro del parque y cubriendo todos los tipos de vegetación. A diferencia de otros carnívoros donde se documentaron avistamientos de más de 20 años atrás, en el caso del cacomixtle se recogieron muchos testimonios de personas que lo han visto muy recientemente y en varias ocasiones y además fue la especie más abundante en las trampas cámara, (41 de 52 registros fotográficos).

\section{Procyon lotor (Linnaeus, 1758)}

Ejemplares examinados. Ninguno. Observaciones. Se tiene el registro de un animal atropellado el 8 de febrero del 2005, a $3 \mathrm{~km}$ de Mineral del Chico, en la localidad 
conocida como Los Corrales. El cuerpo del mapache no se recuperó y solamente se cuenta con las fotografías del animal. Esta especie también se registró en las trampascámara, muy cerca de Los Corrales, lugar donde 14 meses antes se encontró el animal atropellado. Se obtuvieron cinco fotografías entre el 23 y el 25 de abril del 2006 y todas ellas fueron tomadas alrededor de las 20:00 hrs. El mapache fue uno de los animales que menos gente identificó en el PNEC, solamente tres de las 15 personas entrevistadas reconocieron a este animal. No obstante fue relativamente fácil registrar al mapache mediante las trampas-cámara, pero se desconoce el estado que guardan sus poblaciones en el parque.

\section{Orden Rodentia}

Familia Sciuridae

Sciurus oculatus (Peters, 1863)

Ejemplares examinados. (2). Se desconoce la localidad exacta dentro del parque, UAEH-M 68 (1), La Compaña, 2,509 (1). Medidas somáticas. De una hembra, LT:530, C:242, P:62, O:34; peso 600 gr. Y un macho, LT:528, C:250, P:67, O:33; peso 580 gr. Observaciones. Los dos animales fueron recogidos por las autoridades del parque a la orilla de la carretera, después de ser atropellados y fueron donados para la realización de este trabajo. La hembra se colectó el 2 de enero del 2006 y el macho el 11 de mayo del mismo año. La coloración de los dos ejemplares varió notablemente, la hembra presentó una coloración dorsal grisácea muy uniforme y un vientre cremoso, mientras que el macho presentó un vientre cremoso, pero dorsalmente exhibió una franja media dorsal de pelos oscuros, casi negros que contrastaron bastante con el resto del dorso. Esta ardilla es la más abundante en el PNEC, durante el trabajo se observó con frecuencia cerca de la carretera y en la mayoría de las localidades muestreadas.

\section{Spermophillus variegatus (Erxleben, 1777)}

Ejemplares examinados. (1) Desarrollo turístico La Gotera, 2,946 m. Medidas somáticas. De una hembra adulta: LT:480, C:220, P:65, O:25; peso 600 gr. Observaciones. El ejemplar examinado fue atropellado el 23 julio del 2006 y fue donado por el personal del parque para su uso en este trabajo. También se observó a un animal joven que conservan como mascota en la Trucha Feliz, pero solamente se tomaron algunas fotografías. Esta ardilla es menos abundante que $S$. oculatus y no fue posible observarla durante el trabajo de campo.

\section{Glaucomys volans (Linnaeus, 1758)}

Ejemplares examinados. (1) El Cedral, 2,785 m. Medidas somáticas. De una hembra adulta: LT:263, C:119, P:31, O:20; peso 83 gr. Observaciones. Esta ardilla anteriormente había sido registrada por Gallina et al. (1974) mediante los testimonios de la gente del lugar, por lo que el animal aquí examinado es uno de los pocos registros confirmados de la ardilla voladora en el estado de Hidalgo (HernándezFlores \& Rojas-Martínez 2010). En este lugar existen encinos de hoja ancha $(Q$. 
rugosa) mezclados con el oyamel, mientras que los arbustos son escasos. La ardilla voladora se colectó mediante una trampa tipo Sherman. Esta ardilla fue reconocida con claridad por menos de la mitad de las personas entrevistadas, algunas personas mencionaban a la ardilla voladora, pero sus descripciones se ajustaban claramente a los de la ardilla arborícola (S. oculatus).

\section{Familia Geomydae}

\section{Thomomys umbrinus (Richardson, 1829)}

Ejemplares examinados. (1). Parque Nacional El Chico, 2,860 m, UAMI, 1136. Medidas Somáticas. De una hembra: LT:195, C:60, P:28, O:07; peso 99.3 gr. Observaciones. El único ejemplar examinado se encuentra depositado en la Colección de Mamíferos de la Universidad Autónoma Metropolitana, Unidad Iztapalapa (UAMI) y fue colectado el 20 de febrero de 1992, se desconoce la localidad exacta, porque en los datos del rótulo sólo se menciona el PNEC y su altitud. La especie se describió por primera vez en El Chico, sin embargo en la actualidad parece ser rara. Se recorrieron los principales valles del parque y durante el trampeo de otros mamíferos no se observaron sus montículos. Algunos de los lugares en donde la observó la gente anteriormente han sido transformados y acondicionados para los turistas. Es probable que aún se encuentre en el parque, sin embargo sus poblaciones seguramente son pequeñas y están muy segregadas.

Familia Muridae

\section{Microtus mexicanus (Saussure, 1861)}

Ejemplares examinados. (4). Campamento Conejos, 2,920 m, UAEH-M 6,11 (3), Campamento Los Cedros, 2,995 m, UAEH-M 5 (1). Tres colectados y uno liberado. Medidas somáticas. De cuatro hembras: LT:128 (109) (138), C:24.5 (21) (27), P:16.5 (12) (20), O:13.5 (10) (18); peso 24.25 (13) (34) gr. Observaciones. Esta especie se colectó en pastizales o cerca de los mismos, rodeados principalmente por árboles de oyamel $(A$. religiosa) y arbustos de Juniperus (taxcal). Este ratón es abundante en los valles del Campamento Conejos, el Centro de Visitantes, Campamento Los Cedros, Llano Grande y en general en los valles que bordean la carretera entre $\operatorname{los} \mathrm{km} 6$ y 8 , al sur del parque. En estos lugares se capturaron u observaron los metoritos y en todos ellos se observaron los caminos y las madrigueras características de estos ratones.

\section{Peromyscus difficilis (Allen, 1891)}

Ejemplares examinados. (13). La Presa, 2,648 m (1), Km. 13 Carretera Mineral del Monte-Mineral del Chico, 2,805 m (5), Campamento Conejos, 2,930 m (3), Presa El Cedral, 2,785 m (4). Dos colectados y 11 liberados. Medidas somáticas. De siete machos: LT:228.1 (211) (239), C:124.7 (110) (135), P:23.7 (23) (26), O:21.6 (20) (23); peso 29.7 gr. (20) (335). Seis hembras: LT:233.7 (213) (243), C:132.3 (120) (139), P:24.2 (24) (25), O:21.5 (21) (24); peso 31.75 (28) (35). Observaciones. Este ratón fue el menos abundante de las tres especies de Peromyscus. Se colectó en ambientes rocosos, junto con $P$. levipes y $P$. melanotis. 
Peromyscus levipes (Merriam, 1898)

Ejemplares examinados. (104). La Presa, 2,648 m (6), Campamento Dos Aguas, 2,568 m (41), Campamento Conejos, 2,930 m (4), Km 13 Carretera Mineral del Monte-Mineral del Chico, 2,805 m (14), Presa El Cedral, 2,785 m (26), La Estanzuela, 2,741 m (10), Km 2 Carretera Mineral del Monte-Mineral del Chico (3). Se colectaron 16 animales y se liberó el resto. Medidas somáticas. De 53 machos: LT:197.53 (147) (222), C:100.47 (43) (130), P: 23.58 (21) (26), O:19.38 (14) (21); peso 21.7 (13) (33). Cincuenta y un hembras: LT:205.16 (157) (232), C:105.49 (130) (65), P:23.2 (15) (26), O:19.35 (17) (21); peso 25.92 gr (16) (41). Observaciones. Fue el roedor más abundante durante el trabajo y comprende más del $40 \%$ de los Peromyscus capturados. Se encuentra bien distribuido en el parque, cubriendo los tipos de vegetación más representativos de acuerdo a su extensión, que son el bosque de Abies y de Abies-Quercus.

\section{Peromyscus melanotis (J.A. Allen \& Chapman, 1897)}

Ejemplares examinados. (15). Campamento Conejos, 2,930 m. Cuatro colectados y once liberados. Medidas somáticas. De 11 machos: LT:142.7 (127) (153), C:54.5 (31) (67), P:19.7 (18) (21), O:17.5 (15) (29); peso 17.9 gr. (12) (21). Cuatro hembras: LT:138.5 (135) (140), C:49.1 (40) (56), P:19.2 (19) (20), O:16.7 (15) (18); peso 16.2 gr (15) (17). Observaciones. Este ratón es nuevo registró para el PNEC, fue la especie de Peromyscus más pequeña que se capturó y una de las menos abundantes, solamente fue más abundante que Peromyscus difficilis. En los individuos colectados de P. melanotis no es tan clara la mancha oscura en la base de las orejas, aunque sí se observa una franja de coloración más obscura en la región media dorsal, que son dos características diagnósticas para la especie (Ceballos \& Galindo 1984; Ramírez-Pulido 1969). Peromyscus melanotis se colectó en zonas abiertas con pastizales y con menos frecuencia dentro del bosque. Junto con esta especie también se colectó $M$. mexicanus, $R$. megalotis y R. sumichrasti. En abril del 2005 se capturó un macho que tenía una larva parásita de mosca (colmoyote) cerca del ano.

\section{Reithrodontomys megalotis (Baird, 1858)}

Ejemplares examinados. (2). Campamento Conejos, 2,920 m (1), km 2 Carretera Mineral del Monte-Mineral del Chico (1). Dos colectados. Medidas somáticas. De una hembra LT:134, C:79, P:19, O:15, peso 10 gr. Un macho LT:160, C:86, P:18.5, O:16, peso 14 gr. Observaciones. La especie es nueva para el PNEC, anteriormente no se había registrado ningún ratón del género Reithrodontomys. Estos ratones se recolectaron únicamente en zonas abiertas, en pastizales rodeados por bosque de oyamel. La hembra del Campamento Conejos se recolectó en abril del 2005, junto a M. mexicanus y P. melanotis. El macho se colectó en agosto del mismo año en una ambiente similar ubicado en el km 2 de la Carretera Mineral del Monte-Mineral del Chico. 
Reithrodontomys sumichrasti (Saussure, 1861)

Ejemplares examinados. (4). Campamento Dos Aguas, 2,568 m (2), 300 al N. del Campamento Conejos, 2,872 m (2). Tres liberados y uno colectado. Medidas somáticas. De tres machos: LT:168, C:93, P:19, O:16; peso $10 \mathrm{gr}$, LT:174, C:97, P:13, O:15; peso 12 gr y LT:170, C:106, P:17, O:15; peso 15 gr. Una hembra: LT:168, C:88, P:17, O:17; peso 14 gr. Observaciones. Este pequeño ratón es nuevo registro para El Chico. A diferencia de $R$. megalotis que se colectó en valles $R$. sumichrasti se capturó al interior del bosque de Abies, junto con P. melanotis y P. levipes.

Orden Lagomorpha

Familia Leporidae

Sylvilagus floridanus (Allen, 1890)

Ejemplares examinados. (2). Km. 13 Carretera Mineral del Monte-Mineral del Chico, 2,805 (1), km 14, Carretera Mineral del Monte-Mineral del Chico, 2,805 (1). Observaciones. No se tomaron en cuenta las medidas de los conejos examinados, debido a que ambos fueron atropellados y el cuerpo se encontraba muy maltratado. No fue posible preparar las pieles, sin embargo por las dimensiones de los animales y la coloración se identificaron como $S$. floridanus. El conejo fue identificado en el parque por todas las personas entrevistadas, también se observó en varias ocasiones durante el trabajo de campo, sin embargo no fue posible capturar ningún animal vivo.

AGRADECIMIENTOS. A los compañeros del área de mamíferos del Laboratorio de Ecología de Poblaciones que ayudaron en el trabajo de campo y autoridades del parque por las facilidades otorgadas. El trabajo es resultado de los proyectos "Desarrollo de patente para determinar el grado de perturbación ambiental en bosques templados cercanos a Pachuca, Hidalgo, México" (PAI-06/07/034A-UAEH) y "Diversidad biológica del estado de Hidalgo" (FOMIX-HGO-2006-C01-43761).

\section{LITERATURA CITADA}

Álvarez del Toro, M. 1985. ¡Así era Chiapas;. Universidad Autónoma de Chiapas. Tuxtla Gutiérrez, Chiapas, México.

Álvarez, T., S.T. Álvarez \& J. López. 1994. Claves para murciélagos mexicanos. Centro de Investigaciones Biológicas del Noroeste, S. C. y Escuela Nacional de Ciencias Biológicas, Instituto Politécnico Nacional, México D.F. 64 pp.

Álvarez, T., S.T. Álvarez \& M. González. 1997. Localidades típicas de mamíferos terrestres de México. México. D.F. Centro de Investigaciones Biológicas del Noroeste, S. C. y Escuela Nacional de Ciencias Biológicas.

Aranda, J.M. 1981. Rastros de los mamíferos silvestres de México. Manual de campo. Instituto Nacional sobre investigaciones de Recursos Bióticos, Xalapa, Veracruz. 198 pp.

Aranda, J.M. 2000. Huellas y otros rastros de los mamíferos grandes y medianos de México. CONABIO, Instituto de Ecología, A.C. Xalapa, Veracruz. 212 pp.

Azcue, M., L. Fernández, J. \& Toussaint. 1940. Catálogo de construcciones religiosas del Estado de Hidalgo. Secretaria de Hacienda y Crédito Pública.

Ceballos, G \& C. Galindo. 1984. Mamíferos silvestres de la Cuenca de México. Limusa. México. 299 pp. 
Ceballos, G., J. Arrollo-Cabrales, R.A. Medellín \& Y. Domínguez. 2005. Lista Actualizada de los mamíferos de México. Revista Mexicana de Mastozoología, 9:21-71.

Cervantes, F., G. Matamoros \& I. Martínez. 1995. Mamíferos silvestres de la unidad de evaluación y monitoreo de la biodiversidad "Ing. Luis Macías Arellano", San Cayetano, Estado de México. Anales del Instituto de Biología UNAM, Serie Zoología, 66:233-239.

Córdova, A. \& J. Rogel. 1998. Usos de la fauna silvestre en el Parque Nacional El Chico, Hidalgo. Tesis Ingeniero en Planeación y Manejo de los Recursos Naturales Renovables. México. Universidad Autónoma Chapingo, Chapingo. 74 pp.

Coronel-Arellano, H. 2004. Inventario de la mastofauna terrestre: el caso del Rancho Santa Elena, Huasca de Ocampo, Hidalgo. Tesis Licenciatura. Centro de Investigaciones Biológicas. Universidad Autónoma del Estado de Hidalgo. Pachuca, Hidalgo, México. 90 pp.

Durán, A. \& A. Larios, 2001. Ordenamiento Ecológico Territorial Estado de Hidalgo. INEGI.

Galindo, C., S. Franco, M. Flores, C. Lazcano, S. Franco \& R. Cortés. 1988. Plan de Manejo Parque Nacional El Chico. Secretaría de Desarrollo Urbano y Ecología. Pachuca, Hidalgo. 55 pp.

Gallina, M., R. González, R. Moutal \& G. Tello. 1974. Bases para la Reestructuración del Parque Nacional "El Chico" Hidalgo, México. Tesis Licenciatura, Facultad de Ciencias. UNAM. México D.F. 114 pp.

González, R. \& F. Cervantes. 2005. Microtus mexicanus. Pp 659-660. In: Ceballos, G. \& G. Oliva (Eds). Los mamíferos silvestres de México. Conabio. Fondo de Cultura Económica. México D.F.. 986 pp.

Hall, E.R. 1981. The mammals of North America. 2da ed. Vol. 1 and 2, John Wiley and Sons, New York.

Hernández-Flores, S.D. \& Rojas-Martínez, A.E. 2010. Nuevos registros para la ardilla voladora (Glaucomys volans) en el estado de Hidalgo, México. Acta Zoológica Mexicana (n.s.). 26 (2):465-468.

INE-SEMARNAT. 2000. Áreas Naturales Protegidas de México con Decretos Federales (1899-2000). SEMARNAP, PNUD (Programa de las Naciones Unidas para el Desarrollo. México. 395 pp.

IUCN 2008. 2008 IUCN Red List of Threatened Species. Www.iucnredlist.org. Downloaded on 03 november 08 .

López-Wilchis, R. \& J. López. 1999. Los Mamíferos de México Depositados en Colecciones de Estados Unidos y Canadá. Vol. 2. Universidad Autónoma Metropolitana. Unidad Iztapalapa. México D.F.

Medellín, R., H. Arita \& O. Sánchez. 1997. Identificación de los murciélagos de México. Clave de Campo. Num. 2. Asociación Mexicana de Mastozoología A. C. México. 83 pp.

Morales-García, 2007. Ensamblaje de mamíferos terrestres en un bosque templado en áreas bajo diferente manejo forestal, en Huasca de Ocampo, México. Tesis Licenciatura. Centro de Investigaciones Biológicas, Universidad Autónoma del Estado de Hidalgo. México. 56 pp.

Moreno, C. 2001. Métodos para medir biodiversidad. M\&T-Manuales y Tesis SEA. Vol. 1. Zaragoza. $84 \mathrm{pp}$.

Navarro-Frías, J., N. Gonzales \& S.T. Álvarez-Castañeda. 2007. Los mamíferos silvestres de Milpa Alta, Distrito Federal: lista actualizada y consideraciones para su conservación. Acta Zoológica Mexicana (n.s.), 23(3):103-124.

Peña, A., D. Smith \& C. Echegaray. 1998. Conservación. Pp 183-210. In: La diversidad biológica de México. CONABIO, México, D.F.

Ramírez-Pulido, J. 1969. Contribución al estudio de los mamíferos del Parque Nacional Lagunas de Zempoala, Morelos, México. Anales del Instituto de Biología UNAM, Serie Zoología, 40:252-290.

Ramírez-Pulido J. \& A. Castro. 1990. Bibliografía reciente de los mamíferos de México: 1983-1988. Universidad Autónoma Metropolitana, Unidad Iztapalapa, México D.F. 120 pp. 
Ramírez-Pulido J. \& A. Castro. 1994. Bibliografía reciente de los mamíferos de México: 1989-1993. Universidad Autónoma Metropolitana, Unidad Iztapalapa, México D.F. 216 pp.

Ramírez-Pulido J., A., Castro, M. Armella \& A. Salame. 2000. Bibliografía reciente de los mamíferos de México: 1994-2000. Universidad Autónoma Metropolitana, Unidad Iztapalapa, México, D.F. 280 pp.

Ramírez-Pulido J., M. Britton, A. Perdomo \& A. Castro. 1986. Guía de los mamíferos de México: referencias hasta 1983. Universidad Autónoma Metropolitana, Unidad Iztapalapa, México, D.F. $720 \mathrm{pp}$.

Romero, F. 2005. Lynx rufus. Pp 362-364. In: Ceballos, G. \& G. Oliva (Eds). Los mamíferos silvestres de México. Conabio. Fondo de Cultura Económica. México. 986 pp.

Sánchez, O. \& G. Oliva. 2005. Reithrodontomys megalotis. Pp 784-785. In: Ceballos, G. y G. Oliva (Eds). Los mamíferos silvestres de México. Conabio. Fondo de Cultura Económica. México. 986 pp.

SEMARNAT. 2002. Norma Oficial Mexicana NOM-059-ECOL-2001, Protección ambiental- Especies nativas de México de flora y fauna silvestres- Categorías de riesgo y especificaciones para su inclusión, exclusión o cambio- Lista de especies en riesgo. Diario Oficial de la Federación, Miércoles 22 de Marzo.

Soto, M., L. Vázquez \& C. Lazcano. 2006. Programa de conservación y manejo Parque Nacional El Chico, México. CONANP. México. 236 pp.

Villavicencio, M., B. Pérez, \&. Marmolejo \& F. Pérez. 1992. Programa de Manejo del Parque Nacional El Chico. Universidad Autónoma del Estado de Hidalgo. Pachuca, Hidalgo. 422 pp.

Wilson, D. \& D. Reeder. (Eds). 2005. Mammals species of the world, a taxonomic and geographic reference. 3erd ed. Smithsonian Institution Press. Waschington, D.C.

Zavala, E. 1995. Encinos Hidalguenses. Universidad Autónoma Chapingo. División de Ciencias Forestales. México. 133 pp. 


\section{ANEXO I. \\ Lista actualizada de los mamíferos del PNEC}

Muestra su estatus de conservación según la IUCN y la Norma Oficial Mexicana 059, el tipo de vegetación en donde se registró y las especies reportadas por primera vez en el parque

\begin{tabular}{|c|c|c|c|c|c|}
\hline Especie & IUCN & TEN & NOM & VEG & NVO \\
\hline Didelphis virginiana & $\mathrm{LC}$ & $\bar{A}$ & & Baq, Bq, P & \\
\hline Dasypus novemcinctus & $\mathrm{LC}$ & A & & $\mathrm{Baq}$ & \\
\hline Cryptotis mexicana & $\mathrm{LC}$ & $\mathrm{D}$ & Pr* & $\mathrm{Ba}, \mathrm{Baq}$, & $\mathrm{X}$ \\
\hline Sorex saussurei & $\mathrm{LC}$ & $\mathrm{E}$ & Pr* & $\mathrm{Ba}$ & $\mathrm{X}$ \\
\hline Dermanura azteca & $\mathrm{LC}$ & ? & & $\mathrm{Bq}, \mathrm{Bq}, \mathrm{Bpq}$ & $\mathrm{X}$ \\
\hline Corynorhinus mexicanus & NT & $\mathrm{D}$ & & $\mathrm{Bpq}$ & \\
\hline Corynorhinus towsendii & $\mathrm{LC}$ & $\mathrm{E}$ & & $\mathrm{Bpq}$ & $\mathrm{X}$ \\
\hline Eptesicus fuscus & $\mathrm{LC}$ & A & & $\mathrm{Ba}$ & $\mathrm{X}$ \\
\hline Lasiurus cinereus & $\mathrm{LC}$ & $?$ & & $\mathrm{Ba}$ & $\mathrm{X}$ \\
\hline Myotis californicus & $\mathrm{LC}$ & $?$ & & $\mathrm{Ba}, \mathrm{Bc}$ & $\mathrm{X}$ \\
\hline Myotis velifer & $\mathrm{LC}$ & E & & $\mathrm{Ba}, \mathrm{Bc}$ & $\mathrm{X}$ \\
\hline Urocyon cinereoargenteus & $\mathrm{LC}$ & $\mathrm{E}$ & & $\mathrm{Ba}, \mathrm{Baq}, \mathrm{Bc}, \mathrm{Bj}$ & \\
\hline Lynx rufus & LC & E & & $\mathrm{Ba}^{*}$ & \\
\hline Mustela frenata & LC & E & & $\mathrm{Ba}$ & \\
\hline Conepatus leuconotus & $\mathrm{LC}$ & $\mathrm{D}$ & & Baq & \\
\hline Mepthitis macroura & $\mathrm{LC}$ & A & & $\mathrm{Ba}^{*}$ & \\
\hline Spilogale gracilis & $\mathrm{LC}$ & D & & $\mathrm{Ba}^{*}$ & $\mathrm{X}$ \\
\hline Bassariscus astutus & $\mathrm{LC}$ & $?$ & & $\mathrm{Ba}, \mathrm{Baq}, \mathrm{Bc}, \mathrm{Bj}$ & \\
\hline Procyon lotor & $\mathrm{LC}$ & A & & $\mathrm{Baq}$ & \\
\hline Glaucomys volans & LC & $\mathrm{E}$ & A & Baq & $\mathrm{X}$ \\
\hline Sciurus oculatus & $\mathrm{LC}$ & $\mathrm{D}$ & $\operatorname{Pr}$ & $\mathrm{Ba}, \mathrm{Baq}, \mathrm{Bc}$ & \\
\hline Spermophilus variegatus & $\mathrm{LC}$ & $\mathrm{E}$ & & $\mathrm{Baq}$ & \\
\hline Thomomys umbrinus & $\mathrm{LC}$ & ? & & & \\
\hline Microtus mexicanus & $\mathrm{LC}$ & $?$ & & $\mathrm{Ba}, \mathrm{P}$ & \\
\hline Peromyscus levipes & $\mathrm{LC}$ & ? & & $\mathrm{Ba}, \mathrm{Baq}$ & \\
\hline Peromyscus difficilis & $\mathrm{LC}$ & $\mathrm{E}$ & & $\mathrm{Ba}, \mathrm{Baq}$ & \\
\hline Peromyscus melanotis & $\mathrm{LC}$ & ? & & $\mathrm{P}$ & $\mathrm{X}$ \\
\hline Reithrodontomys megalotis & $\mathrm{LC}$ & $\mathrm{E}$ & & $\mathrm{P}$ & $\mathrm{X}$ \\
\hline Reithrodontomys sumichrasti & $\mathrm{LC}$ & $\mathrm{E}$ & & $\mathrm{Ba}, \mathrm{Baq}$ & $\mathrm{X}$ \\
\hline Sylvilagus floridanus & LC & $\mathrm{A}$ & & $\mathrm{Ba}$ & \\
\hline
\end{tabular}

IUCN: categoría de acuerdo a la Unión Internacional para la Conservación de la naturaleza $(E X=$ extinta, $E W=$ extinta en estado silvestre, $\mathrm{CR}=$ críticamente amenazada, $\mathrm{EN}=$ en peligro, $\mathrm{VU}=$ vulnerable, $\mathrm{NT}=$ cercanamente amenazada y $\mathrm{LC}=$ en menor riesgo. TEN: tendencia poblacional según la IUCN $(\mathrm{A}=$ aumento, $\mathrm{E}=\mathrm{estable}, \mathrm{D}=$ descenso y ?= sin datos). NOM: estado de conservación que les confiere la SEMARNAT (NOM-059-Ecol-2001). Se conserva el estado de especie rara de la NOM-059-Ecol-1994. A= amenazada, $\mathrm{E}=$ probablemente extinta en el medio silvestre, $\mathrm{P}=\mathrm{en}$ peligro de extinción, $\mathrm{Pr}=$ sujeta a protección especial y $\mathrm{R}=$ rara. VEG: tipo de vegetación en donde se registraron las especies (los asteriscos indican que se registraron únicamente mediante las entrevistas). $\mathrm{Ba}=$ bosque de Abies, Baq= bosque de Abies-Quercus, $\mathrm{Bc}=$ bosque de Cupresus, $\mathrm{Bj}=$ bosque de Juniperus, $\mathrm{Bq}=$ bosque de Quercus, Bpq= bosque de Pinus-Quercus y P= pastizal. NVO: representa un nuevo registro para el PNEC. 